\title{
The potential approaches to achieve channel reciprocity in FDD system with frequency correction algorithms
}

\author{
Yantao Han, Jiqing Ni \\ Research Institute of Communication Technology \\ Beijing Institute of Technology University (BIT) \\ Beijing, China
}

E-mail: \{autumnwind,njq\}@bit.edu.cn

\author{
Gaoke du \\ Huawei Company \\ Beijing, China \\ E-mail: \{Gaoke.du\}@huawei.com
}

\begin{abstract}
Channel reciprocity is an inherit feature of time division duplex (TDD) system, which is widely used to get uplink (UL)/downlink (DL) channel knowledge from DL/UL channel measurements without additional feedback. While in frequency division duplex (FDD) system, the transmitter usually obtains the DL channel state information (CSI) through a variety of feedback strategies in order to enhance the system performance. However, the impacts of feedback overhead and delay on Multiple-Input-Multiple-Output (MIMO) performance can't be ignored. The paper focuses on the potential approaches to realize FDD channel reciprocity so as to reduce the feedback channel overhead. Compared with TDD system, UL and DL are allocated by different frequencies in FDD system thus the frequency correction is needed to achieve channel reciprocity. The paper presents the performance analysis and comparison of typical frequency correction algorithms, especially the algorithms based on the long-term statistical channel characteristics, which mainly include the channel covariance matrix, the channel space-time correlation, and the direction of arrival (DoA). The potential application scenario for each approach is presented as conclusion.
\end{abstract}

Keywords-receprocity; FDD; frequency correction; $D O A$; covariance matrix; Spatio-Temporal Correlation

\section{INTRODUCTION}

A Multiple-Input-Multiple-Output (MIMO) system is equipped with antenna arrays at both transmitter and receiver. MIMO technology has attracted substantial attention in wireless communications, because it can achieve higher spectral efficiency and link reliability or diversity. MIMO has been adopted as a fundamental technique both for single-user and multi-user in IMT-Advanced. In the downlink, the system performance can be significantly enhanced through the use of scheduling, beam-forming and power allocation techniques. To achieve beam-forming, the Base Station (BS) transmitter must be informed with the channel state information (CSI) of the DL. This has motivated the proposal of many approaches to provide the channel state information at the transmitter (CSIT) more efficiently.

There are roughly two categories to provide CSIT depending upon the chosen duplex scheme for the wireless network. In the case of TDD system, it is proposed to obtain CSIT by using the reciprocity of the UL and DL channels, in order to avoid the resource consume brought by feedback channel. However, in FDD system, UL and DL are allocated with different frequencies and hence the channel realizations can be safely assumed independent of each other. Therefore, a dedicated feedback is used, in which the user conveys the information of the estimated DL channel back to the BS, due to the lack of the channel reciprocity. Moreover, there are also many strategies proposed on how to use a limited feedback channel efficiently while providing the BS with exploitable CSIT. Recently, several interesting strategies have attracted attention to the channel reciprocity in FDD system.

Since UL and DL are allocated by different frequencies in FDD system, the frequency correction is needed to achieve channel reciprocity. In [1], the base station (BS) reuses the spatial (angular) information derived during UL reception, but a prerequisite for this algorithm is that the spatial channel characteristics at the UL and DL frequencies are similar. Different channel sounding campaigns is processed in [2]-[5] to discuss whether the DL channel information can be obtained by the UL channel information in FDD system, but the conclusions seem to be contradictory. Therefore, further discussion through the estimation and calculation of the field measurement is presented in [6], which proves that the UL and DL channel spatial information is relevant, and the UL channel spatial information can be used for DL beam-forming. Similarly, in [7], when the UL and DL frequency interval is set to be $200 \mathrm{MHz}$ of FDD system, which means that the UL and DL channel fading are not related, the DOAs of them are roughly the same. Reference [8]-[10] introduce the methods that DL covariance matrix is transformed by the use of UL channel covariance matrix. In addition, 3GPP proposals [11][17] have also made a discussion among various companies. It is shown in [11] that DL covariance matrix can be generated utilizing UL covariance matrix at $\mathrm{BS}$, and the transformation matrix is provided. In [18], both beam-forming and MUMIMO results for a FDD system with actual field measurements at $3.5 \mathrm{GHz}$ are provided. The estimation of both temporal and spatial DL correlation using UL correlation is investigated in [20], but is not considered in a joint way. Therefore, an improved algorithm is presented in [21], which the conversion from UL to DL is treated jointly on the temporal and the spatial correlation. 
In this paper, we present the performance analysis and comparison of typical frequency correction algorithms, especially the algorithms based on the long-term statistical channel characteristics, which mainly include the channel covariance matrix, the channel space-time correlation, and the direction of arrival (DoA).

\section{CHANNEL MODEL}

The channel model is critical for the simulation of using channel reciprocity in FDD system. We consider the channel models according to the geometry of array.

Suppose that both the transmit and receive arrays are uniform linear array (ULA) and there are k scatters (within one cluster) between the transmitter and receiver, one physical MIMO channel model for this cluster is

$$
\mathrm{H}=\sum_{k=1}^{K} \beta_{k} \mathrm{a}_{R}\left(\phi_{R, k}\right) \mathrm{a}_{T}^{H}\left(\phi_{T, k}\right)
$$

Where $\beta_{k}$ is the path gain for the $k$ th scatter, $\phi_{T, k}$ and $\phi_{T, k}$ are AOD and AOA, respectively.

Fig 1 shows the diagram of equidistant linear antenna for receiving the signal. It is assumed that the electromagnetic wave transmits through a long distance. When it reaches the space antenna array, the reached wave can be regarded as a plane wave. The same assumption can also be employed by the transmitting signal.

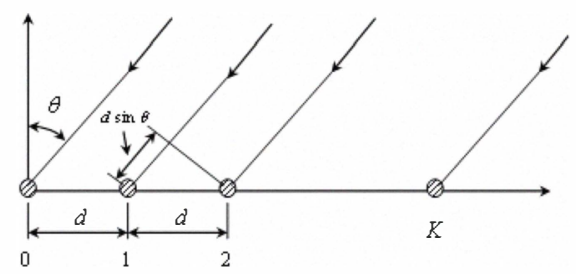

Figure 1. uniform linear array (ULA) receiving signal diagram

Array response vectors at both sides are described as:

$$
\begin{aligned}
\mathrm{a}_{T}\left(\phi_{T, k}\right) & =\left[\begin{array}{c}
1 \\
\exp \left(-j 2 \pi \theta_{T, k}\right) \\
\vdots \\
\exp \left(-j 2 \pi(M-1) \theta_{T, k}\right)
\end{array}\right] \\
\mathrm{a}_{R}\left(\phi_{R, k}\right) & =\left[\begin{array}{c}
\exp \left(-j 2 \pi \theta_{R, k}\right) \\
\vdots \\
\exp \left(-j 2 \pi(N-1) \theta_{R, k}\right)
\end{array}\right]
\end{aligned}
$$

Where $\theta_{T, k}=d_{t} \sin \left(\phi_{T, k}\right) / \lambda$ and $\theta_{R, k}=d_{r} \sin \left(\phi_{R, k}\right) / \lambda$, the wavelength is $\lambda, d_{t}$ and $d_{r}$ are respectively the distance between the transmit and receive array.

The equation (1) can be written as matrix form:

$$
\mathrm{H}=\mathrm{A}_{R} \mathrm{H}_{P} \mathrm{~A}_{T}^{H}
$$

Where $\mathrm{A}_{R}=\left[\mathrm{a}_{R}\left(\phi_{R, 1}\right), \ldots, \mathrm{a}_{R}\left(\phi_{R, K}\right)\right]$ is $\mathrm{M}$ by $\mathrm{K}$ matrix, $\mathrm{A}_{T}=\left[\mathrm{a}_{T}\left(\phi_{T, 1}\right), \ldots, \mathrm{a}_{T}\left(\phi_{T, K}\right)\right] \quad$ is $\mathrm{N}$ by $\mathrm{K}$ matrix, and $\mathrm{H}_{p}=\operatorname{diag}\left(\beta_{1}, \ldots, \beta_{K}\right)$ is diagonal matrix.

\section{ANALYSIS OF FREQUENCY CORRECTION ALGORITHM}

Since UL and DL are allocated by different frequencies in FDD system, the frequency correction is needed to achieve channel reciprocity. This section analyses all kinds of algorithms based on different long-term statistical characteristics.

\section{A. Frequency Correction Based on DOA}

Recently, several contributions have discussed whether the DL channel state information can be obtained by the UL channel state information in FDD system ${ }^{[1]-[5]}$. Reference [6] summarized the potential ways of the above documents, and provided a more comprehensive analysis. Assume the UL channel frequency with $1935 \mathrm{MHz}$ and the DL channel frequency with $2125 \mathrm{MHz}$, the difference of the dominant UL and DL DOA is obtained as

$$
\varphi_{\text {dom, diff }}\left(f_{U L}, f_{D L}\right)=\varphi_{\text {dom }}\left(f_{U L}\right)-\varphi_{\text {dom }}\left(f_{D L}\right)
$$

Though a great deal of measurements, the probability of the DOA difference is

$$
\begin{aligned}
& \mathrm{P}\left\{\varphi_{\text {dom,diff }} \in\left(-0.5^{\circ}, 0.5^{\circ}\right)\right\}=0.18 \\
& \mathrm{P}\left\{\varphi_{\text {dom,diff }} \in\left(-1.5^{\circ}, 1.5^{\circ}\right)\right\}=0.46 \\
& \mathrm{P}\left\{\varphi_{\text {dom, diff }} \in\left(-2.5^{\circ}, 2.5^{\circ}\right)\right\}=0.62 \\
& \mathrm{P}\left\{\varphi_{\text {dom, diff }} \in\left(-4.5^{\circ}, 4.5^{\circ}\right)\right\}=0.81
\end{aligned}
$$

Where $\mathrm{P}\{\boldsymbol{\bullet}\}$ indicates the probability of the values. In general, the dominant DOAs in UL and DL show only a minor deviation. Therefore, the utilization of the dominant DOA estimated during UL reception for DL beam-forming purpose is reasonable.

The system performance between the non-equal gain beamforming and beam-forming using UL DOA through channel reciprocity is compared in [14]. The DL covariance matrix utilized from UL channel DOA can be described as

$$
\mathbf{R}_{D L}=p \mathbf{a}_{D L}\left(\theta_{\max }\right) \mathbf{a}_{D L}^{H}\left(\theta_{\max }\right)
$$

Where $\theta_{\max }$ is the UL DOA. This algorithm is proposed based on the similitude of DOA between the UL and DL channel. Compared with non-equal gain beam-forming, the algorithm makes a small loss in the system performance. But in MU-MIMO applications, performance loss will be increased, since DOA loses the important spatial information.

In addition, the accurate estimate of DOA from the UL data is the key to this algorithm. 


\section{B. Frequency Correction Based on Covariance Matrix}

The methods for the UL to DL information transformation based on the covariance matrix were provided in [8]-[19], which further realize the frequency correction. In FDD system, the typical algorithms for UL to DL information transformation are based on the channel long-term information (e.g. covariance matrix), because the long-term statistic covariance matrix changes much more slowly than the coherence time and bandwidth of the channel. Therefore, a covariance matrix is valid on both UL and DL frequency bands. Even though the duplex distances are large, we can use the frequency correction techniques to improve the accuracy.

In [8], UL to DL covariance matrix transformation techniques based on Minimum Mean Square Error (MMSE) is introduced. And then, Minimum Variance Distortion-less Response (MVDR) filter is presented in [9], in order to obtain DL covariance matrix based on UL covariance matrix. The transformation process is shown as Fig 2. .

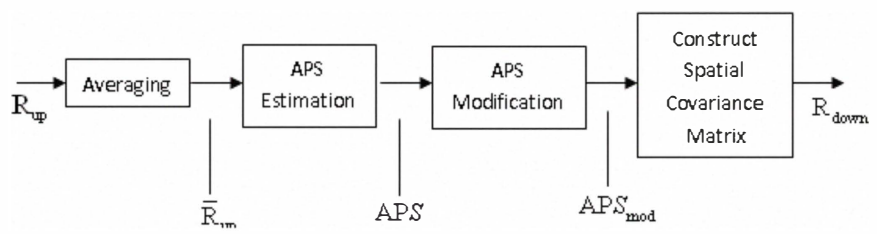

Figure 2. UL to DL Covariance Matrix Transformation

Due to the effects of the angular spread and multi-paths, there are errors between the presumed and the actual UL steering vectors. Reference [10] has proposed a scheme to correct the errors, and in comparison, the system performance is enhanced. And this method is further discussed in [12], considering the impacts of the antennas on the channel reciprocity. The main issues included the impact of the antenna response on DL covariance matrix, and the affect of correlation between antennas on the measurement of UL covariance matrix In addition, reference [12] proposed an algorithm to obtain DL covariance matrix from UL covariance matrix by frequency transformation matrix.

Assuming a uniform linear array (ULA), UL/DL covariance matrix at the base station (BS) can be shown as

$$
\boldsymbol{R}=\int_{g} p(\phi) \boldsymbol{a}(\phi) \boldsymbol{a}^{H}(\phi) d \phi
$$

where $p(\phi)$ is the ray power density at direction of arrival/departure angle $\phi$, and $\boldsymbol{a}(\phi)$ is the antenna array response (steering vector). In the condition of ULA, the antenna array response in the UL and DL of direction $\phi$ is obtained as

$\mathrm{a}_{U L}(\phi)=\left[1, \exp \left(-j 2 \pi \frac{d}{\lambda} \frac{f_{U L}}{f_{0}} \sin (\phi)\right), \cdots, \exp \left(-j 2 \pi \frac{d}{\lambda} \frac{f_{U L}}{f_{0}}(M-1) \sin (\phi)\right)\right]^{H}$ and

$\mathrm{a}_{D L}(\phi)=\left[1, \exp \left(-j 2 \pi \frac{d}{\lambda} \frac{f_{D L}}{f_{0}} \sin (\phi)\right), \cdots, \exp \left(-j 2 \pi \frac{d}{\lambda} \frac{f_{D L}}{f_{0}}(M-1) \sin (\phi)\right)\right]^{H}$ where $d$ is the antenna separation [in meters], $\lambda$ is the wavelength of the carrier [in meters], and $M$ the number of array antennas. The ULA is designed for the carrier frequency $f_{0}, f_{D L}$ and $f_{U L}$ are the DL and UL carrier frequencies respectively.

If the frequency duplex is large, in order to estimate DL channel covariance matrix accurately, a frequency transformation matrix is introduced as

$$
\mathrm{a}_{D L}(\phi)=\mathrm{T}(\phi) \mathrm{a}_{U L}(\phi)
$$

and the diagonal transformation matrix is

$$
\mathrm{T}(\phi)=\operatorname{diag}\left(1, e^{-j 2 \pi \frac{d}{\lambda} \frac{f_{U L}-f_{D L}}{f_{0}} \sin (\phi)}, \cdots, e^{\left.-j 2 \pi \frac{d}{\lambda} \frac{f_{U L}-f_{D L}}{f_{0}}(M-1) \sin (\phi)\right)}\right)
$$

Then downlink covariance estimate can be obtained as

$$
\widehat{\mathrm{R}}_{D L}=\mathrm{T}(\phi) \mathrm{R}_{U L} \mathrm{~T}^{H}(\phi)
$$

By estimating the dominating DOA in the UL, for example, $\phi_{\max }$, we can improve the estimate of $\hat{\mathrm{R}}_{D L}$.

In [19], it's assumed that the uniform circular array (UCA) for UL and DL, the same algorithm is used to obtain (11).

\section{Frequency Correction Based on Spatio-Temporal Correlation}

In general, space-time pre-processing at the base station, such as beam-forming or channel pre-coding, can profit from the knowledge of the DL spatio-temporal correlation properties. Compared with methods above considering the spatial correlation only, reference [20] investigates the estimation of both temporal and spatial DL correlation using an UL correlation estimate. However, the frequency transformation is not considered in a joint way. On the basis of [20], reference [21] presented an improved algorithm, in which the conversion of UL to DL correlation is treated jointly for the temporal and the spatial correlation. The results show that the correlation between UL and DL is related to the ratio between UL and DL carrier frequency. Consequently, the DL correlation can gain easily from the UL correlation.

Assuming the transmitter with $n_{T}$ antennas and the receiver with 1 antenna, with $\mathrm{S}$ discrete scatters surrounding the mobile user, the channel impulse response can be obtained as

$$
h(n)=\sum_{s=1}^{S} \alpha_{s} \exp \left\{j\left(2 \pi f_{D, s} n T_{s l}+\varphi_{s}\right)\right\} a\left(\theta_{s}\right)
$$

Where $T_{s l}$ is the time slot duration, $\alpha_{s}$ is the signal amplitude, $\theta_{s}$ is the angle of arrival as seen from the base station, $f_{D, s}$ is the Doppler shift and $\varphi_{s}$ a random phase shift of the $\mathrm{S}^{\text {th }}$ scatter, respectively. The vector $a\left(\theta_{s}\right)$ is the steering vector of a uniform linear array (ULA).

The spatio-temporal correlation matrix is defined as follows

$$
R(m)=E\left\{h(n) h^{H}(m+n)\right\}
$$

Where the $(i, j)$ element of the matrix $R$ is 
$r([i-j], n)=[R(n)]_{i, j}=\sum_{s=1}\left|\alpha_{s}\right|^{2} \exp \left\{j\left(2 \pi\left[\frac{D_{i e}}{\lambda}(i-j) \sin \theta_{s}-f_{D, s} n{ }_{s l}\right]\right\}\right.$

It can be seen that the correlation depends on the carrier frequency. Assume the UL frequency $f_{u}$ and the DL frequency $f_{l}$, then the UL correlation function can be given as

$$
r_{u}(m, n)=\sum_{s=1}^{S}\left|\alpha_{s}\right|^{2} \exp \left\{j\left(2 \pi\left[\frac{D_{i e}}{\lambda_{u}} m \sin \theta_{s}-f_{D, s, u} n T_{s l}\right]\right\}\right.
$$

Similarly, DL correlation function $r_{d}(m, n)$ can be gained, with $\lambda_{u}=\frac{c_{0}}{f_{u}} f_{D, s, u}=v_{0} f_{u} / c_{0} \cos \beta_{s}$, UL and DL correlation can be seen to depend on each other via

$$
r_{d}(m, n)=r_{u}\left(m \cdot k_{g}, n \cdot k_{g}\right)
$$

Where $k_{g}=\frac{f_{d}}{f_{u}}$ is the relative duplex gap.

In addition, a fast calculation algorithm based on Fourier coefficient is also proposed in [21].

\section{SIMULATION RESULTS}

Due to the duplex frequency distances between UL and DL in FDD systems, the UL and DL covariance matrix are different. In the simulation, the impact of covariance matrix mismatch is investigated and the related simulations are provided by taking the adaptive codebook as an example. In addition, the effects brought by the antenna correlation are also analyzed. The simulation parameters are listed in TABLE I.

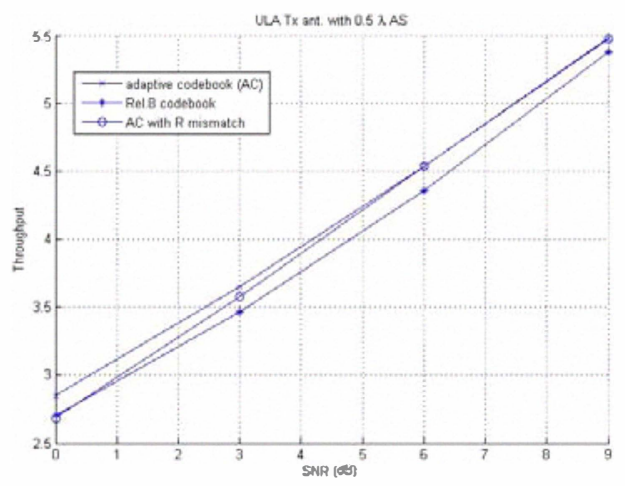

Figure 3. Performance comparison of ULA with $0.5 \lambda$ AS

TABLE I. SIMULATION PARAMETER

\begin{tabular}{|c|c|}
\hline Bandwidth & $5 \mathrm{M}$ \\
\hline UE Speed $(\mathrm{km} / \mathrm{h})$ & 3 \\
\hline Pre-coding type & Subbands (5PRB) and Wideband \\
\hline Feedback type & 2 Subbands or Wideband feedback \\
\hline $\begin{array}{c}\text { Antenna } \\
\text { configuration }\end{array}$ & $\begin{array}{c}4 \times 2 \\
0.5 \text { and } 4 \text { wavelength antenna spacing at } \\
\text { eNodeB } \\
0.5 \text { wavelength antenna spacing ULA or cross } \\
\text { polarized antennas at UE }\end{array}$ \\
\hline $\begin{array}{c}\text { Polarization } \\
\text { antenna }\end{array}$ & ULA and Cross polarized \\
\hline RS density & $\begin{array}{c}\text { DL: } 6 \text { CRS/antenna/PRB } \\
\text { UL: } 30 \text { SRS/antenna/subband/5ms }\end{array}$ \\
\hline $\begin{array}{c}\text { Channel } \\
\text { estimation }\end{array}$ & Ideal \\
\hline CQI estimation & Ideal \\
\hline Receiver & MRC/MMSE \\
\hline $\begin{array}{c}\text { Baseline } \\
\text { codebook }\end{array}$ & Rel.8 codebook \\
\hline MIMO mode & SU-MIMO with Rank-1 transmission \\
\hline
\end{tabular}

\begin{tabular}{|c|c|}
\hline Parameter & Assumption \\
\hline Channel model & SCM UMa \\
\hline $\begin{array}{c}\text { Carrier } \\
\text { Frequency }\end{array}$ & DL: $2.85 \mathrm{GHz}, \mathrm{UL}: 2.51 \mathrm{GHz}$ \\
\hline $\begin{array}{c}\text { Number of } \\
\text { Subcarriers }\end{array}$ & 512 \\
\hline
\end{tabular}

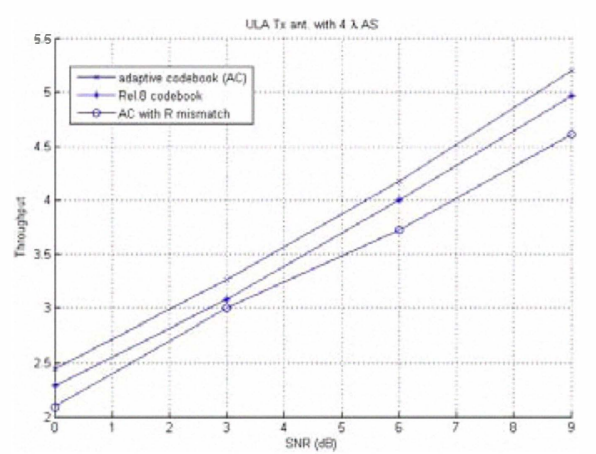

Figure 4. Performance comparison of ULA with $4 \lambda$ AS

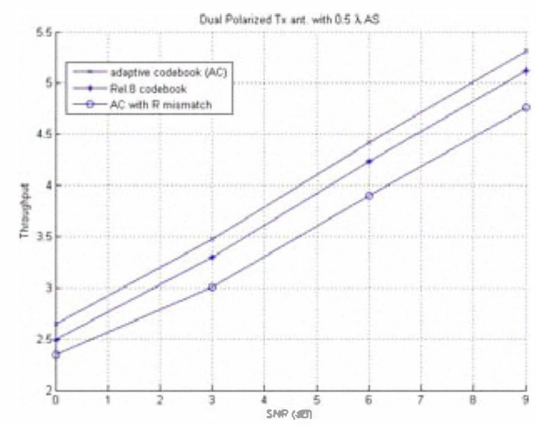

Figure 5. Performance comparison of dual-polarized antenna array with $0.5 \lambda$ AS 


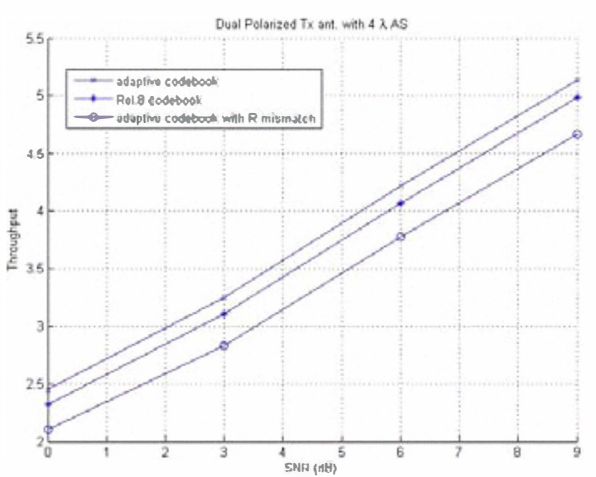

Figure 6. Performance comparison of dual-polarized antenna array with $4 \lambda$ AS

The performance comparison based on ULA with $0.5 \lambda$ antenna spacing is shown in Fig 3. As shown in the result, UL transmission based DL covariance estimation works quite well. The impact of $\mathrm{R}$ mismatch is very limited and can be negligible.

Performances with ULA with $4 \lambda$ and dual-polarized antenna array are evaluated, which were shown in Fig.5, Fig6 and Fig.7. Even though the channel of ULA with $4 \lambda$ is still relatively correlated, the performance degradation however becomes quite significant. When dual-polarized antenna array is assumed, the performance degradation becomes even severer

As can be seen from the simulation, compared with the traditional feedback form, using the channel reciprocity to estimate DL covariance matrix based on UL covariance matrix has a performance loss inevitably, which is due to a variety of factors, such as the antennas correlation. When the correlation is poor, it is not appropriate to use channel reciprocity. Certainly, there are maybe other factors, for instance frequency duplex distance, antenna configuration propagation scenarios, and so on. However, the channel reciprocity does have advantages in reducing the system overhead, it can be inferred that as long as we design a practical system, in which a certain loss conditions can be accept but also accept a certain loss conditions, it can be considered to apply FDD channel reciprocity.

\section{CONCLUSION}

The paper presents the performance analysis and comparison of typical frequency correction algorithms, especially the algorithms based on the long-term statistical channel characteristics, which mainly include the channel covariance matrix, the channel space-time correlation, and the direction of arrival (DoA). Due to the duplex frequency distances between UL and DL in FDD systems, to achieve a DL beam-former from UL channel state information, we usually focus on the parameters or statistics of the propagation channel that remain almost the same between UL and DL. The performance of frequency correction algorithm based on DoA strongly relied on the accuracy of DOA estimation using UL signal, and may not be adopted for the scenarios with angular spreading and distributed scatter. However, the algorithm based on the transformation from UL channel information to
DL channel information, does not make any assumption about spatial distribution of scatters, and it can transpose second order statistics of the channel from UL to DL frequency. Moreover, the performance of this algorithm is affected by the antenna array topology, the appropriate antenna array can enhance system performance. The algorithm based on the Spatio-Temporal correlation considered both the spatial channel characteristics and the time characteristics. However, it is computationally exhaustive.

\section{REFERENCES}

[1] A. Kuchar, M. Tangemann, E. Bonek, "A Real-Time DOA-Based Smart Antenna Processor", IEEE Transactions on Vehicular Technology, to be published.

[2] L. Bigler, H. P. Lin, S. S. Jeng and G. Xu,"Experimental Direction of Arrival and Spatial Signature Measurements at $900 \mathrm{MHz}$ for Smart Antenna Systems", Proc.IEEE VTC'95, Vol. 1, pp. 55-58, Chicago,USA (1995).

[3] K. I. Pedersen, P. E. Mogensen, F.Fredriksen, "Joint-Directional Properties of UL and DL Channel in Mobile Communications", Electronic Letters, Vol.35, No. 16, pp. 1311-1312, 5. August 1999.

[4] B. H. Allen, "Smart Antennas for High Data Rate FDD Wireless Links", Dissertation, University of Bristol, Bristol, UK, April 2001

[5] S. E. Foo, et. al., "Frequency Dependency of Spatial-Temporal Characteristics of UMTS FDD links", COST 273 TD(02)027,Guildford, UK, January 2002.

[6] Klaus Hugl, Kimmo Kalliola and Juha Laurila, "Spatial Reciprocity of UL and DL Radio Channels in FDD Systems", COST 273 TD(02) 066Espoo, Finland,2002/May/30-31

[7] S. E. Foo and M. A. Beach, "UL based DL Beam-forming in UTRA FDD", COST 273 TD(02)104 Lisbon, Portugal, September 19-20, 2002

[8] T Astet, P. Forstert, L. Fety, S. Mayrargue, "DL beam-forming avoiding DOA estimation for cellular mobile communications", Acoustics, Speech and Signal Processing, 1998

[9] Klaus Hugl, Juha Laurila and Ernst Bonck, "DL beam-forming for frequency division duplex systems", Advanced Signal Processing for Communications, 1999

[10] B. K. Chalise, L. Haering, and A. Czylwik, "Robust UL to DL spatial covariance matrix transformation for DL beamforming," in IEEE International Conference on Communications, 2004, vol. 5, Jun.2004, pp 3010-3014

[11] R1-092737, "On CSI feedback for IMT-Advanced Fulfilling CoMP Schemes", Ericsson

[12] R1-100053, "On channel reciprocity for enhanced DL Multi-Antenna transmission", Ericsson

[13] R1-100118, "Discussion on long-term channel reciprocity in FDD", Samsung

[14] R1-100205, "Use of UL Covariance for DL MIMO in FDD", Motorola

[15] R1-100529, "Channel Reciprocity for LTE-A FDD", ZTE

[16] R1-100255, "DL covariance estimation based on reciprocity", Huawei

[17] R1-100853, "Channel reciprocity in FDD systems including systems with large duplex distance", Ericsson

[18] R1-093984, "Use of UL Covariance for DL MIMO in FDD", Motorola

[19] Batu K. Chalise, Lars Hiring, and Andreas Czylwik, "UL to DL Spatial Covariance Matrix Transformation Methods for DL Beam-forming of a UMTS-FDD System", Vehicular Technology Conference, 2004

[20] M. Jordan, G. Ascheid, and H. Meyr, "Prediction of DL SNR for opportunistic beamforming," IEEE Wireless Communications and Networking Conference WCNC 2007, 2007.

[21] Markus Jordan, Xitao Gong, and Gerd Ascheid, "Conversion of the Spatio-Temporal Correlation from UL to DL in FDD Systems", IEEE Communications Society WCNC 2009, 2009 